\section{Hematuria after endoscopic ultrasound-guided fine needle aspiration of a renal tumor in von Hippel-Lindau disease}

A 35-year-old man presented at our hospital with weight loss and diarrhea. Computed tomography (CT) enteroclysis was carried out for evaluation of the small bowel to investigate the diarrhea. The scan revealed bilateral renal cysts with complex renal masses, as well as multiple pancreatic cysts and a mass in the genu ( $\bullet$ Fig. 1). The patient was suspected as having von Hippel-Lindau (VHL) disease, which was confirmed after a CT brain revealed a cerebellar hemangioblastoma.

Endoscopic ultrasound (EUS) evaluation of the pancreas showed multiple pancreatic cysts between the head and the tail ( $\bullet$ Fig. 2) with an $18 \times 12 \mathrm{~mm}$, well-defined hyper-echoic mass in the genu. Both kidneys were studded with cysts. In addition, a hyper-echoic mass $(12 \times 15 \mathrm{~mm})$ was seen in the right kidney.

We carried out EUS-guided fine needle aspiration (FNA) of the pancreatic mass, followed by the right renal mass ( $\bullet$ Fig. 3; - Video 1).

However, shortly afterward, the patient passed blood-stained urine, and hematuria was confirmed with a dipstick. As it was mild, the patient was managed conservatively, and the hematuria resolved several hours later. The renal aspirate revealed a renal cell carcinoma (RCC), while the pancreatic aspirate revealed a neuroendocrine tumor. The patient was offered surgery, but he declined.

VHL disease is an autosomal dominant condition characterized by tumors involving multiple organs [1]. We report the first case of EUS-guided FNA of a renal mass in VHL disease resulting in hematuria. This complication has never been documented before, and there are only four reports of

\section{Video 1}

Endoscopic ultrasound (EUS)-guided fine needle aspiration (FNA) of the right renal mass carried out from the second portion of the duodenum: a hyperechoic lesion (centre) is seen originating in the right kidney (lower left).

Smaller cysts are present too.

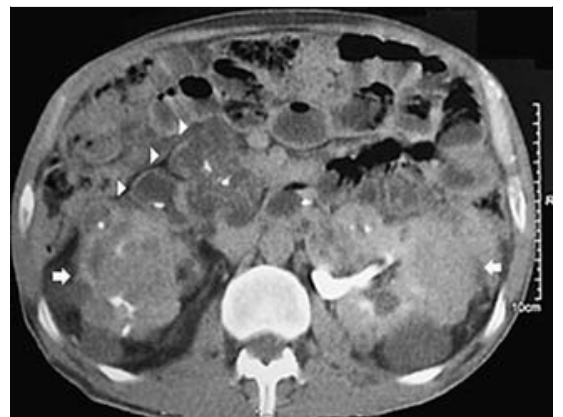

Fig. 1 Computed tomography (CT) scan in a middle-aged patient with weight loss and diarrhea: multiple cysts (arrowheads) are present in the pancreas, extending from the genu to the distal body. Note also the bilateral renal masses (arrows).

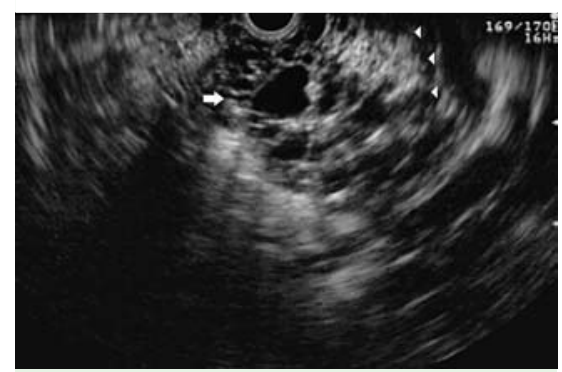

Fig. 2 Linear endoscopic ultrasound of the pancreas carried out from the stomach: the sagittal view of the pancreatic body shows cysts of varying sizes (arrow). Minimal pancreatic parenchyma is visible (arrowheads).

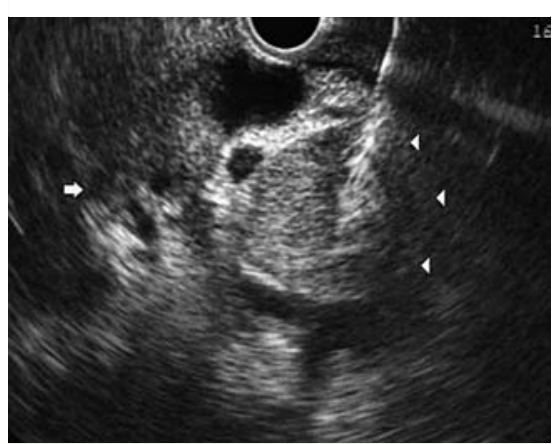

Fig. 3 Endoscopic ultrasound (EUS)-guided fine needle aspiration (FNA) of a renal mass carried out from the duodenum: a hyperechoic mass in the right kidney (arrowheads) was punctured with a 22-G needle. Several smaller renal cysts are also present (arrow). uncomplicated EUS-guided FNA of renal masses [2-5]. The risks associated with percutaneous biopsy of renal lesions include hematoma, hematuria, pneumothorax, and needle tract seeding. Endoscopists should be aware of these complications because a renal mass may be accessible only by traversing through the parenchyma. Our case also illustrates the usefulness of EUS in the evaluation of both pancreas and kidneys in VHL disease.

\section{Endoscopy_UCTN_Code_CPL_1AL_2AF}

\section{Competing interests: None}

\section{S. Lakhtakia, E. Wee, R. Gupta,}

S. Anuradha, R. Kalpala, A. Monga,

S. Arjunan, D. N. Reddy

Asian Institute of Gastroenterology,

Hyderabad, India

\section{References}

1 Shuin T, Yamasaki I, Tamura K et al. HippelLindau disease: molecular pathological basis, clinical criteria, genetic testing, clinical features of tumors and treatment. Jpn J Clin Oncol 2006; 36 (6): 337-343

2 Farrell JJ, Brugge WR. EUS-guided fine-needle aspiration of a renal mass: an alternative method for diagnosis of malignancy. Gastrointest Endosc 2002; 56 (3): 450-452

3 De Witt J, Gress FG, Levy MJ et al. EUS-guided FNA aspiration of kidney masses: a multicenter U.S. experience. Gastrointest Endosc 2009; 70 (3): $573-578$

4 Artifon EL, Lopes RI, Kumar A et al. Endoscopic ultrasound facilitates histological diagnosis of renal cell cancer. J Endourol 2008; 22 (11): 2447-2450

5 Eloubeidi MA, Tamhane A, Jhala $N$ et al. Agreement between rapid onsite and final cytologic interpretations of EUS-guided FNA specimens: implications for the endosonographer and patient management. Am J Gastroenterol 2006; 101 (12): 2841 - 2847

\section{Bibliography}

DOI $10.1055 / \mathrm{s}-0030-1256682$

Endoscopy 2012; 44: E133

(c) Georg Thieme Verlag KG Stuttgart · New York . ISSN 0013-726X

\section{Corresponding author}

\section{S. Lakhtakia}

Asian Institute of Gastroenterology

6-3-661 Somajiguda

Hyderabad - 500082

India

Fax: +91-40-23324255

sandeep@lakhtakia.com 